\title{
Si Doped GaAs (001) Films
}

S. Zhen

Shanghai Jiao Tong University, 800 Dongchuan Rd., Shanghai 200240, China.

The short research of optimization of the growth method to obtain p-type GaAs (001) layers using $\mathrm{Si}$ as the dopant was reported in this work. Atomic force microscopy was used to analyze the surface morphology and low-temperature photoluminescence also used to confirm the p-type of the layers.

The growth of high quality p-type layers has always been a problem by using MOVPE or MBE system. At the beginning of the growth, beryllium has been commonly used for the p-type doping on GaAs(001) layers [1-5]. In addition, carbon has proven to be a good substitute, allowing doping concentration up to $1020 \mathrm{~cm} 23$ with virtually no memory effect, but still there are no reliable carbon for solid-source. An attractive option is to exploit the amphoteric behavior of silicon ( $\mathrm{Si}$ ), with the advantage that one single element could be used to obtain $\mathrm{n}$ - and p-type layers in bipolar devices. Despite the advantages presented by these orientations (piezoelectric effects and low incorporation of background impurities), the $\mathrm{GaAs}(001)$ orientation is still the favorite one to produce optoelectronic devices due to its two orthogonal cleavage axis. A new growth method was suggested recently [7-11] that allows the use of Si as the p-type dopant with GaAs(001) substrates. A few monolayers of gallium were deposited together with the dopant in the absence of arsenic and later were annealed under an As flux to form the GaAs:Si layers. In these special growth conditions, most of the $\mathrm{Si}$ atoms tend to incorporate into the As sites where they act as acceptors, as confirmed by Hall measurements [7]. This sequence can be repeated hundreds of times to obtain thick GaAs:Si layers with a p character. Unfortunately, the samples grown in that way usually exhibited a low hole mobility due to structural defects created by the peculiar growth mode. In the present work, we report on the morphological and optical properties of such layers and seek for the optimal growth conditions that yield a minimal number of defects.

All the samples were grown in a homemade MOVPE system on top of semi-insulating $\mathrm{GaAs}(001)$ substrates. Initially, a $200 \mathrm{~nm}$-thick GaAs buffer layer was grown under conventional conditions to assure that each sample would start with the same morphological conditions. Two samples with the same thickness $(250 \mathrm{~nm})$ were grown using different amounts of Ga flow and annealing times as: $15 \mathrm{ML}$ of Ga doped Si and $15 \mathrm{sec}$ of As annealing in sample A and $5 \mathrm{ML}$ of $\mathrm{Ga}: \mathrm{Si}$ and $10 \mathrm{sec}$ of As in sample B. The growth temperature was set at $1060^{\circ} \mathrm{C}$ for all the samples during the growth of the doped layer to avoid excessive re-evaporation of arsenic from the GaAs surface. As a reference sample, we grew a GaAs:Si layer under conventional conditions with the same thickness and $\mathrm{Si}$ concentration used for the other samples. The surface of the samples was analyzed with an AFM system in contact mode and Photoluminescence (PL) experiments were carried out at $1.4 \mathrm{~K}$ using conventional lock-in techniques.

Figure 2 shows the AFM images of the 2 samples along the [1-10] direction can be observed and their size and concentration decrease when the amount of $\mathrm{Ga}$ in each cycle is lowered. This is mainly due to the fact that, under this special growth condition, the first Ga layer covers the whole surface while the excess of Ga material forms droplet spread all over the surface that act 
as reservoirs of Ga material in order to form the GaAs:Si layers during the annealing under As flux $[12,13]$.

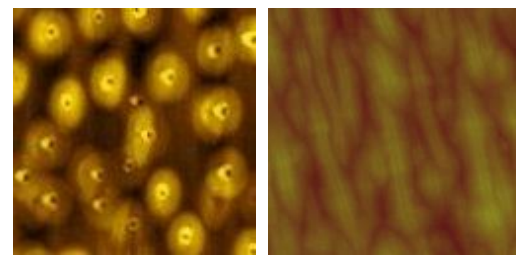

Figure 1. $2 \times 2 \mu \mathrm{m}^{2}$ AFM images of the samples grown in different conditions by MOVPE

It is clear from Fig. 2 that the PL emission depends on the growth conditions which was used to form and dissolve the droplets. When incearsing the $\mathrm{Ga}$ in each growth cycle, it is also necessary to increase the As exposure time to transform the larger quantity of Ga material into a two dimensional GaAs:Si layer. So, the Si atoms that were evaporated with the Ga material and that are not bound to any $\mathrm{Ga}$ atom have more time to eventually enter the $\mathrm{Ga}$ and act as donors. This behavior compensates the sample and shifts the emissions to higher energy levels which was observed.

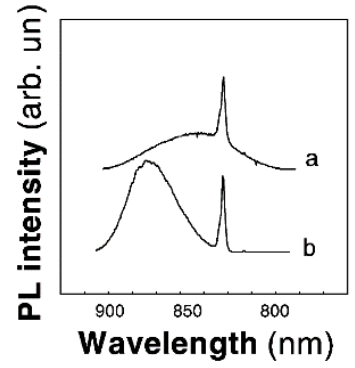

Figure 2. PL spectra measured at $1.4 \mathrm{~K}$ of two samples.

The morphological and optical properties of p-type $\mathrm{GaAs}(001)$ layers doped with Si during was studied and achieved by a new growth method based on the deposition of excess Ga with $\mathrm{Si}$ atoms followed by annealing in an As flux. The AFM results showed that layers with good morphological properties can be obtained by decreasing the amount of Ga:Si. PL also showed the p-type layers and confirmed that the growth cycle to get the best p-type GaAs(001) layers consists of the alternate deposition of $\mathrm{Ga}: \mathrm{Si}$.

[1] M. Ilegems, J. Appl. Phys. 48 (1977) 1278 https://doi.org/10.1063/1.323772

[2] C. Chung et al., Nanoscale Res. Lett. 9 (2014) 338 https://doi.org/10.1186/1556-276X-9-338

[3] C. Chung et al, Electron. Mater. Lett. 10 (2014) 457-460 https://doi.org/10.1007/s13391-

$\underline{013-3202-3}$

[4] H. Nguyen et al., Appl. Phys. Express 5 (2012) 055503

https://doi.org/10.1143/APEX.5.055503

[5] C. Chung et al., Mater. Res. Express 2 (2015) 055505 https://doi.org/10.1088/2053$1591 / 2 / 5 / 055505$

[6] H. W. Yu et al., Jpn. J. Appl. Phys. 51 (2012) 080208 https://doi.org/10.1143/JJAP.51.080208

[7] A.A. Quivy et al, J. Cryst. Growth 206 (1999) 171 https://doi.org/10.1016/S00220248(99)00325-5 
[8] C. Nguyen et al., Electron. Mater. Lett. 10 (2014) 759-762 https://doi.org/10.1007/s13391014-4016-7

[9] C. Chung et al., Electron. Mater. Lett. 10 (2014) 963-967 https://doi.org/10.1007/s13391014-3201-Z

[10] N. Nguyen et al., 10th IEEE International Conference on Semiconductor Electronics (ICSE), 19-21 Sept. (2012) 13266309 DOI: 10.1109/SMElec.2012.6417133

[11] H. Trinh et al., 10th IEEE International Conference on Semiconductor Electronics (ICSE), 19-21 Sept. (2012) 13266425 DOI: 10.1109/SMElec.2012.6417251

[12] T.E. Lamas, A.A. Quivy, Braz. J. Phys. 32 (2002) 399 https://doi.org/10.1590/S010397332002000200043

[13] R.C. Newman, Semicond. Sci. Technol. 9 (1994) 1749 https://doi.org/10.1088/0268$\underline{1242 / 9 / 10 / 001}$ 